CERN-PH-EP-2005-039

12 August 2005

\title{
Measurement of topological muonic branching ratios of charmed hadrons produced in neutrino-induced charged-current interactions
}

\author{
CHORUS Collaboration
}

\begin{abstract}
From 1994 to 1997, the emulsion target of the CHORUS detector was exposed to the wideband neutrino beam of the CERN SPS. In total about 100000 charged-current neutrino interactions were located in the nuclear emulsion target and fully reconstructed. From this sample of events based on the data acquired by new automatic scanning systems, 2013 charm-decay events were selected by a pattern recognition program. They were confirmed as decays through visual inspection. Based on these events, the effective branching ratio of charmed particles into muons was determined to be $\overline{B_{\mu}}=[7.3 \pm 0.8$ (stat) \pm 0.2 (syst) $] \times 10^{-2}$. In addition, the muonic branching ratios are presented for dominating charm decay topologies. Normalization of the muonic decays to chargedcurrent interactions provides $\sigma_{\mu^{-} \mu^{+}} / \sigma_{\mathrm{cc}}=[3.16 \pm 0.34$ (stat) \pm 0.09 (syst) $] \times 10^{-3}$. Selecting only events with visible energy greater than $30 \mathrm{GeV}$ gives a value of $\overline{B_{\mu}}$ that is less affected by the charm production threshold and quasi-elastic $\Lambda_{\mathrm{c}}^{+}$production. Combining this value with the current average of $\overline{B_{\mu}} \times\left|V_{\mathrm{cd}}\right|^{2}$ at the leading order yields the value of $\left|V_{\mathrm{cd}}\right|_{\mathrm{LO}}=0.236 \pm 0.016$.
\end{abstract}




\title{
CHORUS Collaboration
}

\author{
A. Kayis-Topaksu, G. Önengüt \\ Çukurova University, Adana, Turkey \\ R. van Dantzig, M. de Jong, R.G.C. Oldeman ${ }^{1}$ \\ NIKHEF, Amsterdam, The Netherlands \\ M. Güler, U. Köse, P. Tolun \\ METU, Ankara, Turkey \\ M.G. Catanesi, M.T. Muciaccia \\ Università di Bari and INFN, Bari, Italy \\ K. Winter \\ Humboldt Universität, Berlin, Germany ${ }^{2}$ \\ B. Van de Vyver ${ }^{3,4}$, P. Vilain ${ }^{5}$, G. Wilquet ${ }^{5}$
}

Inter-University Institute for High Energies (ULB-VUB) Brussels, Belgium

B. Saitta

Università di Cagliari and INFN, Cagliari, Italy

E. Di Capua

Università di Ferrara and INFN, Ferrara, Italy

S. Ogawa, H. Shibuya

Toho University, Funabashi, Japan

I.R. Hristova ${ }^{6}$, T. Kawamura, D. Kolev ${ }^{7}$, H. Meinhard, J. Panman, A. Rozanov ${ }^{8}$, R. Tsenov ${ }^{7}$,

J.W.E. Uiterwijk, P. Zucchelli ${ }^{3,9}$

CERN, Geneva, Switzerland

J. Goldberg

Technion, Haifa, Israel

M. Chikawa

Kinki University, Higashiosaka, Japan

J.S. Song, C.S. Yoon

Gyeongsang National University, Jinju, Korea

K. Kodama, N. Ushida

Aichi University of Education, Kariya, Japan

S. Aoki, T. Hara

Kobe University, Kobe, Japan

T. Delbar, D. Favart, G. Grégoire, S. Kalinin, I. Makhlioueva

Université Catholique de Louvain, Louvain-la-Neuve, Belgium

A. Artamonov, P. Gorbunov, V. Khovansky, V. Shamanov, I. Tsukerman

Institute for Theoretical and Experimental Physics, Moscow, Russian Federation

N. Bruski, D. Frekers

Westfälische Wilhelms-Universität, Münster, Germany ${ }^{2}$

K. Hoshino, J. Kawada, M. Komatsu, M. Miyanishi, M. Nakamura, T. Nakano, K. Narita, K. Niu,

K. Niwa, N. Nonaka, O. Sato, T. Toshito

Nagoya University, Nagoya, Japan

S. Buontempo, A.G. Cocco, N. D’Ambrosio, G. De Lellis, G. De Rosa, F. Di Capua, G. Fiorillo,

A. Marotta, M. Messina, P. Migliozzi, L. Scotto Lavina, P. Strolin, V. Tioukov

Università Federico II and INFN, Naples, Italy

T. Okusawa

Osaka City University, Osaka, Japan

U. Dore, P.F. Loverre, L. Ludovici, G. Rosa, R. Santacesaria, A. Satta, F.R. Spada

Università La Sapienza and INFN, Rome, Italy

E. Barbuto, C. Bozza, G. Grella, G. Romano, C. Sirignano, S. Sorrentino

Università di Salerno and INFN, Salerno, Italy

Y. Sato, I. Tezuka

Utsunomiya University, Utsunomiya, Japan 
${ }^{1}$ Now at University of Liverpool, Liverpool, UK.

${ }^{2}$ Supported by the German Bundesministerium für Bildung und Forschung under contract numbers 05 6BU11P and 05 7MS12P.

${ }^{3}$ Now at SpinX Technologies, Geneva, Switzerland.

${ }^{4}$ Fonds voor Wetenschappelijk Onderzoek, Belgium.

${ }^{5}$ Fonds National de la Recherche Scientifique, Belgium.

${ }^{6}$ Now at DESY, Hamburg.

${ }^{7}$ On leave of absence and at St. Kliment Ohridski University of Sofia, Bulgaria.

${ }^{8}$ Now at CPPM CNRS-IN2P3, Marseille, France.

${ }^{9}$ On leave of absence from INFN, Ferrara, Italy. 
Introduction

Dimuon production in neutrino charged-current interactions has been studied in several experiments, in particular, CDHS [1], CCFR [2], CHARM [3], CHARM-II [4], NOMAD [5] and NuTeV [6] by means of electronic detectors. In these events, the leading muon is interpreted as originating from the neutrino vertex and the other, of opposite charge, as the decay product of the charmed particle. The study of dimuon events provides information on the strange quark content of the nucleon, the charm mass, and the Cabibbo-Kobayashi-Maskawa (CKM) matrix elements $V_{\mathrm{cd}}$ and $V_{\mathrm{cs}}$. Experiments of this type, however, suffer from the presence of a large background in which the second muon originates from an unrecognized decay of a pion or a kaon rather than from a charm decay. Moreover, the type of charmed particle and its decay topology cannot be precisely identified in these experiments. The overall normalization of the charm production rate in terms of the dimuon rate is given by the average semileptonic branching fraction, defined as the weighted average of the semileptonic branching fractions for individual charmed hadron species with the corresponding charmed fractions as weights.

Compared with the study of dimuon events with electronic detectors, a much lower level of background can be achieved using an emulsion target which provides a sub-micron spatial resolution and, hence, the topological identification of charmed hadron decays. This technique has been applied in the E531 experiment at FNAL to measure the production fractions $f_{D_{i}}$. These data have been combined $[7,8,9]$ with the individual semileptonic branching fractions to yield the probability that charm particles decay semileptonically. However, the statistics accumulated in the E531 experiment was limited to 125 charm events and the result was affected by the poor knowledge of the branching ratio of the $\mathrm{D}^{0}$ into a final state with only neutral particles. Only recently, with the development of automatic scanning devices of much higher speed within CHORUS, it has become possible to study large samples of charm events produced in nuclear emulsions [10].

The inclusive measurement of the semileptonic branching ratio of charmed hadrons based on a smaller sample of events has already been reported in a previous paper [11, 12]. Since then a completely new muon reconstruction algorithm has been implemented with the aim of reducing the systematic error on the measurement. The current analysis is based on the complete sample of 2013 manually confirmed charm events. Taking advantage of the manual measurement of the decay topology, the muonic branching ratio is determined separately on the basis of the number of charged daughters ('prongs') of the charmed particle. The number of events is sufficient to determine the average muonic branching fraction directly from the number of charm events with a secondary muon in the final state, with an uncertainty comparable with that obtained by existing, indirect, measurements.

\section{The experimental apparatus}

The CHORUS detector [13] is a hybrid set-up that combines a nuclear emulsion target with various electronic detectors. The nuclear emulsion is used as target for neutrino interactions, allowing a threedimensional reconstruction of short-lived particles like the $\tau$ lepton and charmed hadrons. The emulsion target, which is segmented into four stacks, has an overall mass of $770 \mathrm{~kg}$, each stack consists of eight modules of 36 plates of size $36 \mathrm{~cm} \times 72 \mathrm{~cm}$. Each plate has a $90 \mu \mathrm{m}$ plastic support coated on both sides with emulsion layers of $350 \mu \mathrm{m}$ thickness. After development, the emulsion thickness diminished on average by a factor of two. This effect is taken into account during track reconstruction. Each stack is followed by three interface emulsion sheets with an emulsion layer of $90 \mu \mathrm{m}$ thickness on both sides of an $800 \mu \mathrm{m}$ thick plastic foil and by a set of scintillating-fibre tracker planes. The interface sheets and the fibre trackers provide accurate predictions of particle trajectories into the emulsion stack for the location of the vertex positions. The accuracy of the fibre tracker prediction is about $150 \mu \mathrm{m}$ in position and $2 \mathrm{mrad}$ in the track angle. The emulsion scanning is performed by fully automatic microscopes equipped with CCD cameras and a readout system, called 'Track Selector' [10]. In order to recognize track segments in the emulsion, a series of tomographic images are taken by focusing at different depths in the emulsion thickness. The digitized images are shifted according to the predicted track angle and then summed. The presence of aligned grains forming a track is detected as a local peak of the grey-level of the summed image. The track-finding efficiency of the Track Selector is higher than $98 \%$ for track slopes less than $400 \mathrm{mrad}[14]$.

The electronic detectors downstream of the emulsion target include a hadron spectrometer that 
measures the bending of the charged particles in an air-core magnet, a calorimeter where the energy and direction of electromagnetic and hadronic showers are measured, and a muon spectrometer which determines the charge and momentum of muons. The calorimeter has a finer-grained upstream part (EM) for the measurement of electromagnetic showers and a coarser-grained downstream part to contain hadronic showers. The hadronic calorimeter, in turn, consists of two parts the so-called HAD1 and HAD2. Together, HAD1 and HAD2 contain 10 scintillator planes and 18 planes of streamer tubes oriented in alternate orthogonal projections. The muon spectrometer is located behind the calorimeter. The calorimeter of 5.2 hadronic interaction lengths filters out nearly all the particles produced by neutrino interactions in the emulsion target except muons with momentum greater than $\sim 1.5 \mathrm{GeV} / c$.

The CHORUS detector was exposed to the wideband neutrino beam of the CERN SPS during the years 1994-97, with an integrated flux of $5.06 \times 10^{19}$ protons on target. The beam, of $27 \mathrm{GeV}$ average energy, consisted mainly of muon neutrinos with a $5 \% \bar{\nu}_{\mu}$ contamination.

\section{Event reconstruction and selection of decay topologies}

The event reconstruction, based on the information from the electronic part of the detector, determines whether an event originates in the emulsion stack. In charged-current events, the primary muon is identified with high efficiency by the reconstruction program and a precise prediction of the impact point of this muon with the downstream plate of the emulsion stack from which it emerges is determined. The algorithm uses the spectrometer, the streamer tubes in the calorimeter, and the fibre tracker to reconstruct this track.

The emulsion scanning of a charged-current event starts with the location of this muon in the most downstream plates using the reconstructed slope and position information. This track is called the 'scan-back track'. The track segments found in these downstream plates are then extrapolated with high precision into the emulsion stack. If found, the scan-back track is then followed upstream from one plate to the next. In each plate, the automatic scanning system records only the most upstream $100 \mu \mathrm{m}$ layer. The disappearance of the scan-back track serves as an indication for the position of the primary vertex. If a scan-back track is not located on two consecutive emulsion plates, the first one is assumed to be the plate containing the vertex. Once the 'vertex plate' is identified, a very fast scanning system, called 'Ultra Track Selector' (UTS) [15], is used to perform a detailed analysis of the emulsion volume around the vertex position, recording, for each event, all track segments within a given angular acceptance. We refer to this type of scanning, originally developed for the DONUT experiment [16], as 'NetScan' data taking [17].

In the CHORUS experiment, the scanning volume is $1.5 \mathrm{~mm}$ wide in each transverse direction and $6.3 \mathrm{~mm}$ along the beam direction, corresponding to eight emulsion plates. This volume contains the vertex plate itself, the plate immediately upstream, acting as a veto for passing through tracks, and the six plates downstream from the vertex plate. The latter six plates act as decay space and are used to detect the tracks of the decay daughters. The scanning area is centred on the extrapolated transverse position of the scan-back track. The angular acceptance corresponds to a cone of $400 \mathrm{mrad}$ half-aperture aligned along the beam direction. At present, about $150000 \nu_{\mu}$ and $\bar{\nu}_{\mu}$ events have been located in emulsion and analysed with this procedure.

The first task of the NetScan event reconstruction in the emulsion is to select, from the large number of recorded track segments, only those belonging to the neutrino interaction under study. The reconstruction algorithm then tries to associate these tracks to common vertices. A detailed description of the algorithms to reconstruct the vertices is given in Ref. [17]. At the end of the procedure, one defines a primary vertex (and its associated tracks) and possibly one or more secondary vertices to which daughter tracks are attached. A further selection is then applied that preserves a high efficiency for decay vertices and rejects most background topologies. In this analysis the same event sample was used as in Ref. [18], where the procedure has been described in detail. From the current sample of 93807 scanned and analysed neutrino-induced charged-current events, these criteria select 2752 events which have been visually inspected ('eye-scan') to confirm the decay topology. A secondary vertex is accepted as a decay if the number of charged particles is consistent with charge conservation and no other activity (Augerelectron or visible recoil) is observed. The result of the visual inspection is given in Table 1. The notation $\mathrm{C} 1, \mathrm{C} 3$, and $\mathrm{C} 5$ is used for decays of charged particles into 1, 3, and 5 charged prongs, respectively, and 
Table 1: The number of charm candidates and the relative number of background events in the various samples

\begin{tabular}{rrr}
\hline Decay topology & Number of charm candidates & Background events \\
\hline C1 & 452 & $43.3 \pm 2.4$ \\
V2 & 819 & $36.6 \pm 3.5$ \\
C3 & 491 & $3.8 \pm 0.2$ \\
V4 & 226 & negligible \\
C5 & 22 & $1.5 \pm 0.1$ \\
V6 & 3 & negligible \\
\hline Total sample & 2013 & 85.2 \\
\hline
\end{tabular}

V2, V4, and V6 for neutral particle decays with 2, 4 and 6 charged prongs, respectively. The purity of the automatic selection is $73.1 \%$.

There is a non-negligible fraction of non-charm events in the manually confirmed sample. This contamination is mainly due to hadronic interactions that fake charm decays (white kinks), and decays of $\Sigma^{ \pm}, K_{\mathrm{s}}^{0}$ and $\Lambda^{0}$. The backgrounds from the decays of the strange particles were estimated using the JETTA [19] MC generator which is based on LEPTO 6.1 [20]. The production rate of the strange particles normalized to the charged-current events was found to be consistent with NOMAD data [21, 22]. The last column of Table 1 shows the number of background events in the charm decay topologies.

\section{$4 \quad$ Reconstruction efficiency}

To estimate the efficiencies of the charmed hadron selection, several Monte Carlo generators were used [23]. The neutrino beam spectrum was simulated using the GBEAM [24] generator. It uses FLUKA98 [25] to describe the interactions of protons with the beryllium target. When the neutrino scatters off a nucleon, different physical mechanisms can produce charmed hadrons. Deep-inelastic scattering processes were simulated using the JETTA generator [19]. Simulation of quasi-elastic charmed baryon production was performed by QEGEN [26]. This generator uses differential cross-sections by Shrock and Lee [27]. For the diffractive production of charmed mesons, the ASTRA [28] generator was used. The simulation of the detector response for each process was performed by a GEANT 3.21 [29] based simulation program. Simulation of the emulsion response can be divided into two parts: event location and event selection. The event location efficiency describes the shortcomings of finding charged-current interactions in emulsion. This efficiency was parametrized by a function of the primary muon momentum and angle. The muon momentum distribution is different for the two samples of CC events, containing and not containing charm. The dependence of the ratio of the location efficiencies as a function of visible energy is not strong, and the average value of the ratio is

$$
\frac{\varepsilon_{\mathrm{loc}}^{\mathrm{charm}}}{\varepsilon_{\mathrm{loc}}^{\mathrm{cc}}}=0.92 \pm 0.02
$$

In order to evaluate the selection efficiency of charmed hadrons in emulsion, realistic conditions have to be reproduced. These were obtained by merging the emulsion data of the simulated events with real NetScan data that do not contain a reconstructed vertex, thus accurately representing the experimental background. The combined data were passed through the same NetScan reconstruction and selection programs as used for real data.

The determination of the muonic branching ratio of charmed hadrons depends on the ratio of the selection efficiency for all decays modes of these hadrons $\left(\mathrm{D}^{0}, \mathrm{D}^{+}, \mathrm{D}_{\mathrm{s}}^{+}\right.$and $\left.\Lambda_{\mathrm{c}}^{+}\right)$and for their specific muonic decay channels. Excited charmed states or other charmed hadrons disintegrate through strong or electromagnetic interactions into these four charmed particles. The difference in the reconstruction 
Table 2: Charm production fractions from a reanalysis of the E531 data (first column) [9] and from the JETTA generator (second column). The fractions take into account the branching fraction of the $\mathrm{D}^{0}$ into final states with all neutrals [18] and they are normalized to the total charm production. The last two columns represent the overall selection efficiency and selection efficiency for decays with a muon in the final state

\begin{tabular}{ccccc}
\hline $\mathrm{D}^{0}$ & $f_{\mathrm{D}^{0}}(\mathrm{E} 531)$ & $f_{\mathrm{D}^{0}}(\mathrm{JETTA})$ & $\varepsilon_{\mathrm{D}^{0}}$ & $\varepsilon_{\mathrm{D}^{0}}^{\mu}$ \\
\hline $\mathrm{V} 0$ & 0.12 & 0.11 & 0 & - \\
$\mathrm{V} 2$ & 0.38 & 0.38 & $0.57 \pm 0.01$ & $0.61 \pm 0.02$ \\
$\mathrm{~V} 4$ & 0.09 & 0.09 & $0.75 \pm 0.01$ & $0.78 \pm 0.02$ \\
\hline
\end{tabular}

\begin{tabular}{ccccc}
\hline $\mathrm{D}^{+}$ & $f_{\mathrm{D}^{+}}(\mathrm{E} 531)$ & $f_{\mathrm{D}^{+}}(\mathrm{JETTA})$ & $\varepsilon_{\mathrm{D}^{+}}$ & $\varepsilon_{\mathrm{D}^{+}}^{\mu}$ \\
\hline $\mathrm{C} 1$ & 0.07 & 0.10 & $0.24 \pm 0.01$ & $0.29 \pm 0.02$ \\
$\mathrm{C} 3$ & 0.07 & 0.12 & $0.53 \pm 0.01$ & $0.56 \pm 0.03$ \\
\hline
\end{tabular}

\begin{tabular}{ccccc}
\hline $\mathrm{D}_{\mathrm{s}}^{+}$ & $f_{\mathrm{D}_{\mathrm{s}}^{+}}(\mathrm{E} 531)$ & $f_{\mathrm{D}_{\mathrm{s}}^{+}}(\mathrm{JETTA})$ & $\varepsilon_{\mathrm{D}_{\mathrm{s}}^{+}}$ & $\varepsilon_{\mathrm{D}_{\mathrm{s}}^{+}}^{\mu}$ \\
\hline $\mathrm{C} 1$ & 0.06 & 0.03 & $0.33 \pm 0.02$ & $0.39 \pm 0.08$ \\
$\mathrm{C} 3$ & 0.05 & 0.04 & $0.70 \pm 0.02$ & $0.77 \pm 0.05$ \\
\hline \multicolumn{5}{c}{} \\
\hline$\Lambda_{\mathrm{c}}^{+}$ & $f_{\Lambda_{\mathrm{c}}^{+}}(\mathrm{E} 531)$ & $f_{\Lambda_{\mathrm{c}}^{+}}(\mathrm{JETTA})$ & $\varepsilon_{\Lambda_{\mathrm{c}}^{+}}$ & $\varepsilon_{\Lambda_{\mathrm{c}}^{+}}^{\mu}$ \\
\hline $\mathrm{C} 1$ & 0.08 & 0.03 & $0.21 \pm 0.02$ & $0.25 \pm 0.05$ \\
$\mathrm{C} 3$ & 0.07 & 0.05 & $0.52 \pm 0.02$ & $0.58 \pm 0.14$ \\
\hline
\end{tabular}

efficiency is taken into account by introducing the following factor for each number of prongs $n$ :

$$
R(n \text {-prong })=\frac{\sum_{D_{i} \rightarrow n} f_{D_{i}} \varepsilon_{D_{i}}}{\sum_{D_{i} \rightarrow n} f_{D_{i}} \varepsilon_{D_{i}}^{\mu}},
$$

where, for example, the sum is taken over $\mathrm{D}_{\mathrm{s}}^{+}, \mathrm{D}^{+}$and $\Lambda_{\mathrm{c}}^{+}$if $n$ is odd. The quantities $f_{D_{i}}$ are the charm production fractions which include the different charm production processes, $\varepsilon_{D_{i}}$ is the selection efficiency for different charm species, $\varepsilon_{D_{i}}^{\mu}$ is the selection efficiency for the charm particles with a muonic decay. For $\mathrm{D}^{0} \rightarrow \mathrm{V} 2$ or V4 (decay of a neutral particle into two or four charged prongs), the $R$-factor becomes just the ratio of these two efficiencies, since $f_{D_{i}}$ cancels out. Also the $R$-factor can similarly be defined for a mixture of different topologies. The first two columns of Table 2 show different estimations of the fractions. In both cases, the same value of the branching ratio of the $\mathrm{D}^{0}$ into final states with all neutral particles is used [18]. One (JETTA) is the model used in the Monte Carlo, the other (E531) is the only direct measurement of the fractions in the literature [7]. These results on the charged charm decay topologies are split here into two different categories, $\mathrm{C} 1$ and $\mathrm{C} 3$. It is worth stressing that the exact values of the production fractions are not very important because the event reconstruction efficiency does not change dramatically from one charm species to the other and the effect is further weakened when taking the sum. For this particular analysis, the ratio $\varepsilon_{D_{i}} / \varepsilon_{D_{i}}^{\mu}$ plays a more important role than the production fractions. Nevertheless, a systematic error on $R$ is introduced as a result of choosing different sets of the fractions $f_{D_{i}}$. In many cases, the errors on $R$ may be neglected since they are small with respect to the other sources.

The selection efficiencies and the production fractions are summarized in Table 2. Figure 1 shows the energy dependence of the selection efficiency when the four charm species are taken together. Two factors determine why at small visible energies the selection is less efficient: the opening angle of charm daughters is larger; the flight path of charm is shorter and thus a secondary track might be attached to the primary vertex. At high energies, a large fraction of charmed hadrons decays near the edge or beyond the fiducial volume. Substitution of the numbers from Table 2 in Eq. (2) gives the estimates of $R$ shown in Table 3. 
Figure 1: Average NetScan selection efficiency for charm as a function of visible energy as obtained with the simulation with the visible energy spectrum of charm events (in arbitrary units) as overlay

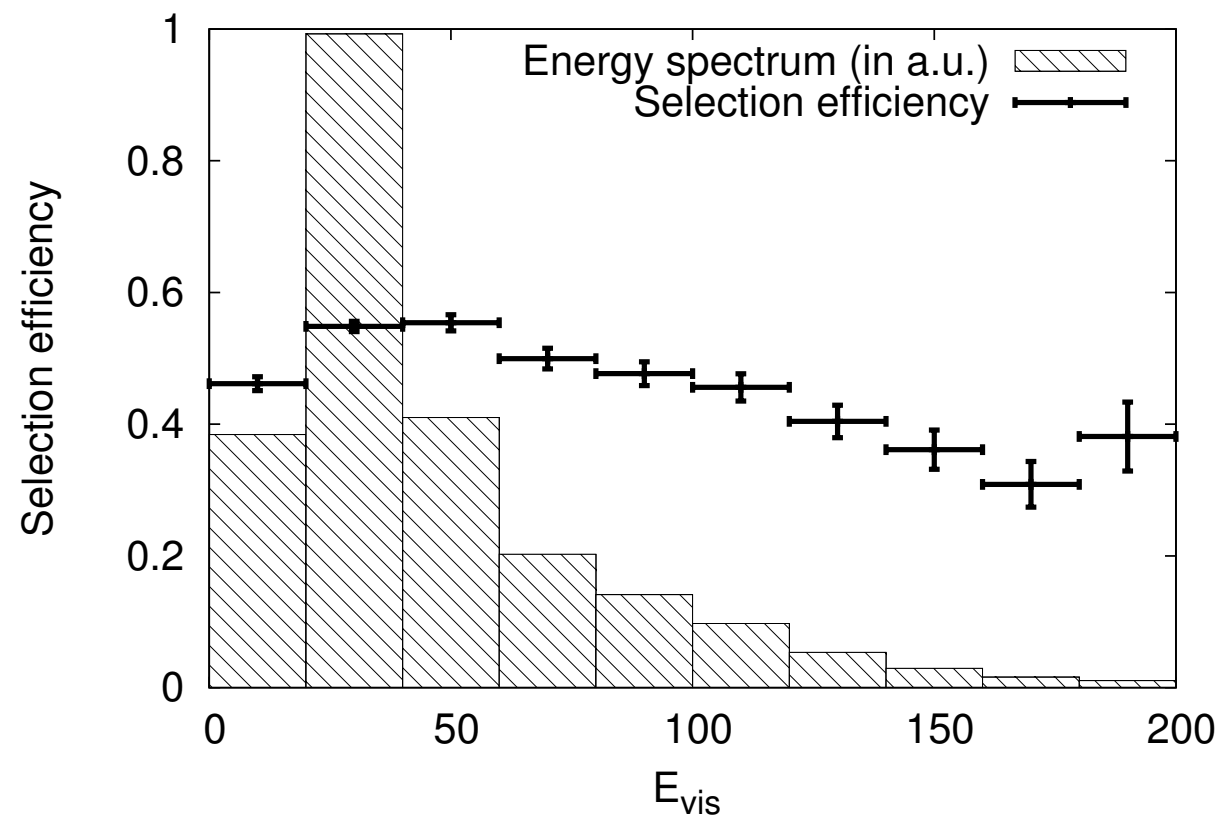

\section{$5 \quad$ Identification of secondary muons}

The identification algorithm is based on the information from the downstream part of the detector: the calorimeter, and the muon spectrometer. The probability for hadrons to reach the muon spectrometer without interaction in the calorimeter is about $5 \%$. In the case of secondary muons, to require a track in the spectrometer would unavoidably lead to a loss in angular acceptance and to a threshold for the muon momentum between $1.5 \mathrm{GeV} / c$ and $2.5 \mathrm{GeV} / c$, depending on the track angle. An algorithm using the range in the calorimeter was developed to sample low-momentum muons. In general, optimal criteria for primary and secondary muons are different because these classes of muons have different kinematical properties and background.

The muon recognition algorithm is track-driven and uses the extrapolation of tracks reconstructed in the emulsion into the calorimeter and the first spectrometer module. A track is assumed to be a muon from a charm decay if it originates from a downstream secondary vertex within the NetScan volume and if it is either matched to a spectrometer track or can be extrapolated with sufficient range as measured by the scintillators or streamer tubes in the calorimeter and the first magnet of the spectrometer. The range

Table 3: The values of the $R$-factor defined in the text for different charm decay topologies and their mixtures. The systematic errors include the uncertainty on charm production fractions and on the branching ratio $\mathrm{D}^{0}$ into neutrals for the inclusive case. The first error quoted is statistical and, when present, the second is the systematic error. The last column shows the relative systematic errors on the muonic branching ratio from the muon identification described in Sec.5.

\begin{tabular}{cll}
\hline Topology & $R$ & $\sigma_{\text {syst }}^{\mathrm{id}}(\%)$ \\
\hline $\mathrm{C} 1$ & $0.83 \pm 0.06 \pm 0.01$ & $4.7 \pm 0.3$ \\
$\mathrm{~V} 2$ & $0.93 \pm 0.02$ & $4.4 \pm 0.2$ \\
$\mathrm{C} 3$ & $0.92 \pm 0.06 \pm 0.01$ & $10.7 \pm 0.8$ \\
$\mathrm{~V} 4$ & $0.96 \pm 0.02$ & negligible \\
$\mathrm{C} 1+\mathrm{C} 3$ & $0.89 \pm 0.04 \pm 0.01$ & $5.6 \pm 0.4$ \\
$\mathrm{~V} 2+\mathrm{V} 4$ & $0.94 \pm 0.01 \pm 0.01$ & $3.6 \pm 0.2$ \\
Inclusive & $0.80 \pm 0.01 \pm 0.01$ & $3.3 \pm 0.1$ \\
\hline
\end{tabular}


Table 4: Number of secondary tracks identified as muons in real data, background normalized to the number of selected tracks, and identification efficiency as obtained from simulation. The errors on the identification efficiencies are determined by the limited Monte Carlo statistics. The identification criteria for V4 sample are explained in the text. In the inclusive case the samples are combined using the weights from Table 2. The last column shows muonic branching ratios for different prong samples and their mixtures. The first error quoted is statistical and the second is the systematic error. The statistical errors include the uncertainty on the number of identified muons and the error on the identification efficiency.

\begin{tabular}{ccccc}
\hline Number of prongs & Selected & Background & $\varepsilon_{\mu}^{\text {id }}, \%$ & $\overline{B_{\mu}}(\%)$ \\
\hline C1 & 20 & 0.8 & $36.0 \pm 3.4$ & $10.8 \pm 2.4 \pm 0.5$ \\
V2 & 34 & 9.8 & $34.5 \pm 1.9$ & $8.3 \pm 1.4 \pm 0.4$ \\
C3 & 17 & 8.4 & $26.4 \pm 2.6$ & $6.1 \pm 1.6 \pm 0.6$ \\
C1+C3 & 37 & 9.2 & $31.7 \pm 3.1$ & $8.6 \pm 1.4 \pm 0.4$ \\
V2+V4 & 36 & 9.8 & $30.1 \pm 1.5$ & $8.1 \pm 1.5 \pm 0.3$ \\
Inclusive & 73 & 19.0 & $30.4 \pm 2.1$ & $7.3 \pm 0.7 \pm 0.2$ \\
\hline
\end{tabular}

condition is only used if there are sufficiently many isolated streamer tube hits found along the track or if the energy deposition in HAD2 is below a threshold value.

There are several sources of background to this muon definition. Those giving the largest contributions are punch-through hadrons and muons from decays of $\pi$ 's and K's that have been matched to emulsion tracks. All other processes give a minor contribution.

The result of applying the criteria described above to tracks emerging from secondary vertices is shown in Table 4 for each decay topology separately. It was verified that none of the background sources presented in Table 1 significantly contributes to the sample with a muon coming from the secondary vertex. Because of the limited statistics in the five-prong and six-prong samples, these events are not included in the table. The identification efficiency as a function of secondary-muon momentum is presented in Fig. 2.

The main source of systematic uncertainty in the muonic branching ratio is the systematic error in the secondary muon identification. The identification method is not very sensitive to changes in the criteria on the matching with muon tracks found in the spectrometer. However, it does depend on the details of the scintillator range estimator, the streamer tubes range estimator, the isolation measured with the streamer tubes, and the energy deposited in the most downstream part of the hadronic calorimeter. An estimate of the systematic error is obtained by a variation of the cuts on the parameters in the secondarymuon identification procedure. For each set of cuts applied to the estimators a value of $B_{\mu}$ is obtained. The variance gives an estimate of the systematic error of the measurement; these uncertainties are given in Table 3.

Another source of the systematic error is the choice of the parameters of the model used in the simulation. The charm quark mass, $m_{\mathrm{c}}$, the choice of the parton distribution functions, GRV94LO[30] and CTEQ3[31], and the relative fraction of the strange sea compared to the down-quark sea, $\kappa$, affect the momentum spectrum of the muons coming from charm decays. The variation limits for $m_{\mathrm{c}}$ and $\kappa$ are consistent with the values of the fit parameters describing the energy dependence of the $\mathrm{D}^{0}$ production cross-section measured with the same sample of events (see Ref. [18]): $m_{\mathrm{c}}=(1.42 \pm 0.08) \mathrm{GeV} / c^{2}$ and $\kappa=0.38 \pm 0.10$. The relative systematic error obtained in this manner corresponding to one standard deviation is equal to $\sigma_{\text {syst }}^{\text {model }}=1.6 \%$. For the V4 sample the tighter requirement that a secondary track be associated with a positively charged spectrometer track was applied in order to optimize the signal to background ratio. This criterion selects two events out of 226. If the spectrometer track is required to have negative charge no events are selected. Background for such events could come from hadronic charm decays with one of the charm daughters decaying muonically. It has also been verified that the muonic background coming from decays of strange particle $\left(\mathrm{K}_{\mathrm{s}}^{0}, \Lambda^{0}\right)$ daughters is small. A Monte Carlo simulation shows that the level of identification background corresponding to this sample is $(3.4 \pm 0.4) \times 10^{-2}$. 


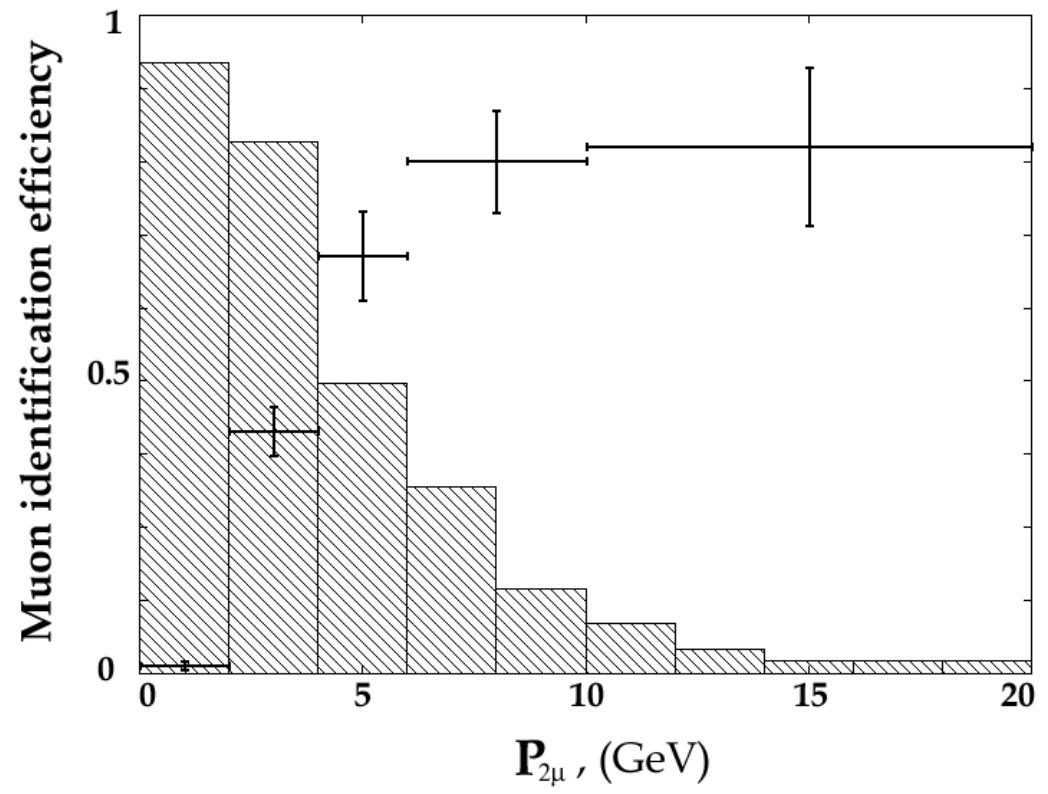

Figure 2: Identification efficiency $\varepsilon_{\mu}^{\text {id }}$ for muons coming from charm and their spectrum as obtained from simulation.

\section{$6 \quad$ Results and conclusions}

$B_{\mu}^{n}$, the muonic branching ratio for each $n$-prong sample, can be written in terms of measurable quantities as

$$
B_{\mu}^{n}=\sum_{i} f_{D_{i}}^{n} B^{n}\left(D_{i} \rightarrow \mu^{+} X\right)=\frac{N_{2 \mu}-N_{\mathrm{bkgr}}}{N \cdot \varepsilon_{\mu}^{\mathrm{id}}} \times R(n \text {-prong }),
$$

where the sum is taken over the contributing charmed hadrons, $N$ is the total number of events in the $n$ prong sample corrected for non-charm background, $N_{2 \mu}$ is the number of tracks identified as secondary muons, $N_{\mathrm{bkgr}}$ is the number of background tracks in $N_{2 \mu}$ from Table 4 , and $\varepsilon_{\mu}^{i d}$ is the secondary muon identification efficiency. The inclusive value of the ratio is calculated as a weighted sum of the ratios per topology

$$
\overline{B_{\mu}}=\sum_{n} f_{C_{n}} \cdot B\left(C_{n} \rightarrow \mu^{+} X\right),
$$

where $C_{n}$ can be $\mathrm{C} 1, \mathrm{~V} 2, \mathrm{C} 3$ or $\mathrm{V} 4$, and $f_{C_{n}}$ are the average fractions obtained from Table 2. Table 4 contains the values of muonic branching ratios as a function of the number of prongs.

The muonic decay branching ratio of the $\mathrm{D}^{0}$ could be obtained by combining the results for the two-prong and four-prong samples and taking into account the branching ratio of the $\mathrm{D}^{0}$ into only neutral particles, $B\left(\mathrm{D}^{0} \rightarrow\right.$ neutrals $)$, mentioned earlier. This yields

$$
B_{\mu}\left(\mathrm{D}^{0}\right)=[6.5 \pm 1.2(\text { stat }) \pm 0.3(\text { syst })] \times 10^{-2},
$$

where the systematic error is determined by the precision of muon identification and $B\left(\mathrm{D}^{0} \rightarrow\right.$ neutrals $)$. This result is in agreement with the value $(6.6 \pm 0.8) \times 10^{-2}$ quoted in Ref. [32].

The limit for the V4 decays could be obtained using the Feldman-Cousins approach [33] with a $90 \%$ confidence level. The result is normalized to the total $\mathrm{D}^{0}$ cross-section:

$$
3.0 \times 10^{-4}<B_{\mu}\left(\mathrm{D}^{0} \rightarrow \mathrm{V} 4\right)<3.4 \times 10^{-3},
$$

to be compared with the existing $90 \%$ C.L. limit $B\left(\mathrm{D}^{0} \rightarrow \mathrm{K}^{-} \pi^{+} \pi^{-} \mu^{+} \nu_{\mu}\right)<1.2 \times 10^{-3}$ [34]. It is also consistent with the value using $\mathrm{D}^{0}$ events in the emulsion, $2.0 \times 10^{-4}<B\left(\mathrm{D}^{0} \rightarrow \mathrm{K}^{-} \pi^{+} \pi^{-} \mu^{+} \nu_{\mu}\right)<$ $1.5 \times 10^{-2}$ at $90 \%$ C.L., which was obtained by counting the number of four-prong charm-decay events with two opposite-charge muons in the final state [35]. 
The average muonic branching ratio for the $\mathrm{C} 1$ and $\mathrm{C} 3$ samples taken together could be reinterpreted as the average branching ratio for charged charm hadrons $\mathrm{D}_{\mathrm{s}}^{+}, \mathrm{D}^{+}$and $\Lambda_{\mathrm{c}}^{+}$(taking into account the small number of $\mathrm{C} 5$ events).

The inclusive muonic branching ratio for the complete sample of charm hadrons is therefore determined to be

$$
\overline{B_{\mu}}=[7.3 \pm 0.8 \text { (stat) } \pm 0.2(\text { syst })] \times 10^{-2} .
$$

Our earlier result, $\overline{B_{\mu}}=[9.3 \pm 0.9$ (stat) \pm 0.9 (syst) $] \times 10^{-2}$ [11], was obtained using a value for $B\left(\mathrm{D}^{0} \rightarrow\right.$ neutrals) from the observed decay channels only [32]. If the new determination for this topological branching ratio obtained in Ref. [18] is used, the previous result is reduced by $\approx 7 \%$. The results are therefore compatible given the entirely different systematic uncertainties of the two measurements. The improvement in the systematic error is significant. Because of the limited statistics of the actual sample, the energy dependence of $\overline{B_{\mu}}$ could be determined in a small number of bins only. Splitting the total sample into three subsamples ( $E_{\mathrm{vis}} \leq 30 \mathrm{GeV}, 30 \mathrm{GeV} \leq E_{\mathrm{vis}} \leq 50 \mathrm{GeV}$ and $50 \mathrm{GeV} \leq E_{\mathrm{vis}}$ ) gives results compatible with each other within one standard deviation.

Strictly speaking, the muonic branching ratio measured in this analysis can not be compared directly with the results from other experiments because the beam spectra are not the same. As additional difference it should be mentioned that the present determination is a direct measurement while in the other experiments $\overline{B_{\mu}}$ was extracted as one of the parameters of a fit to kinematical distributions.

In a similar manner, one can obtain the dimuon cross-section normalized to the charged-current cross-section. In terms of measurable quantities the ratio is

$$
\frac{\sigma_{\mu^{-} \mu^{+}}}{\sigma_{\mathrm{cc}}}=\frac{N_{2 \mu}-N_{\mathrm{bkgr}}}{N_{\mathrm{cc}} \cdot \varepsilon_{\mu}^{\mathrm{id}}} \times \frac{\varepsilon_{\mathrm{loc}}^{\mathrm{cc}}}{\varepsilon_{\mathrm{loc}}^{\text {charm }}} \times \frac{1}{\varepsilon_{\mathrm{rec}}^{\text {charm }}},
$$

where $N_{\mathrm{cc}}$ is the number of charged-current events in a bin, $\varepsilon_{\text {loc }}$ are the location efficiencies from Eq 1 , and $\varepsilon_{\text {rec }}^{\text {charm }}$ is the average event reconstruction efficiency of charm decaying muonically from Table 2 (evaluated to be $\varepsilon_{\text {rec }}^{\text {charm }}=0.57$ ). The result is shown in Fig. 3 as a function of energy. The average value is

$$
\frac{\sigma_{\mu^{-} \mu^{+}}}{\sigma_{\mathrm{cc}}}=[3.16 \pm 0.34 \text { (stat) } \pm 0.09 \text { (syst) }] \times 10^{-3} .
$$

The CCFR experiment has extracted the value for $\left|V_{\mathrm{cd}}\right|$ by combining its study of opposite-sign dimuon events with a determination of $\overline{B_{\mu}}$ based on the E531 data, considering only events with a visible energy above $30 \mathrm{GeV}$ [2]. This approach has been adopted by the particle data group as well [32], even though it is then applied on the average over $\overline{B_{\mu}}\left|V_{\mathrm{cd}}\right|^{2}$ measurements from different experiments with LO and NLO analyses. Such a procedure requires an assumption on the scale uncertainty. To avoid that, we extract the value of $\left|V_{\mathrm{cd}}\right|$ for LO and NLO separately. Averaging the measurements at the leading order by the CDHS, CHARM II and CCFR experiments [9], one would get

$$
\overline{B_{\mu}} \times\left|V_{\mathrm{cd}}\right|^{2}=(0.474 \pm 0.027) \times 10^{-2} .
$$

Selecting events from our sample with visible energy greater than $30 \mathrm{GeV}$ yields a value of

$$
\overline{B_{\mu}}=[8.5 \pm 0.9 \text { (stat) } \pm 0.6 \text { (syst) }] \times 10^{-2} .
$$

Adding the statistical and systematic errors in quadrature, and combining with the value above we find that

$$
\left|V_{\text {cd }}\right|_{\text {LO }}=0.236 \pm 0.016 .
$$

Up to now, there is only one measurement at the next-to-leading order by the CCFR experiment [2]

$$
\overline{B_{\mu}} \times\left|V_{\mathrm{cd}}\right|^{2}=\left[0.534 \pm 0.046 \text { (stat) }{ }_{-0.051}^{+0.025}(\text { syst })\right] \times 10^{-2},
$$

where the last error is from the scale uncertainty. Substitution of our value for $\overline{B_{\mu}}$ and symmetrization of the errors give

$$
\left|V_{\mathrm{cd}}\right|_{\mathrm{NLO}}=0.251 \pm 0.021
$$

Both values should be compared with $0.221<\left|V_{\text {cd }}\right|<0.227$ at $90 \%$ C.L. that is obtained when imposing CKM-matrix unitarity and only three generations [32]. Also they are compatible with our previous measurement [11] $\left|V_{\mathrm{cd}}\right|=0.219 \pm 0.022$. 


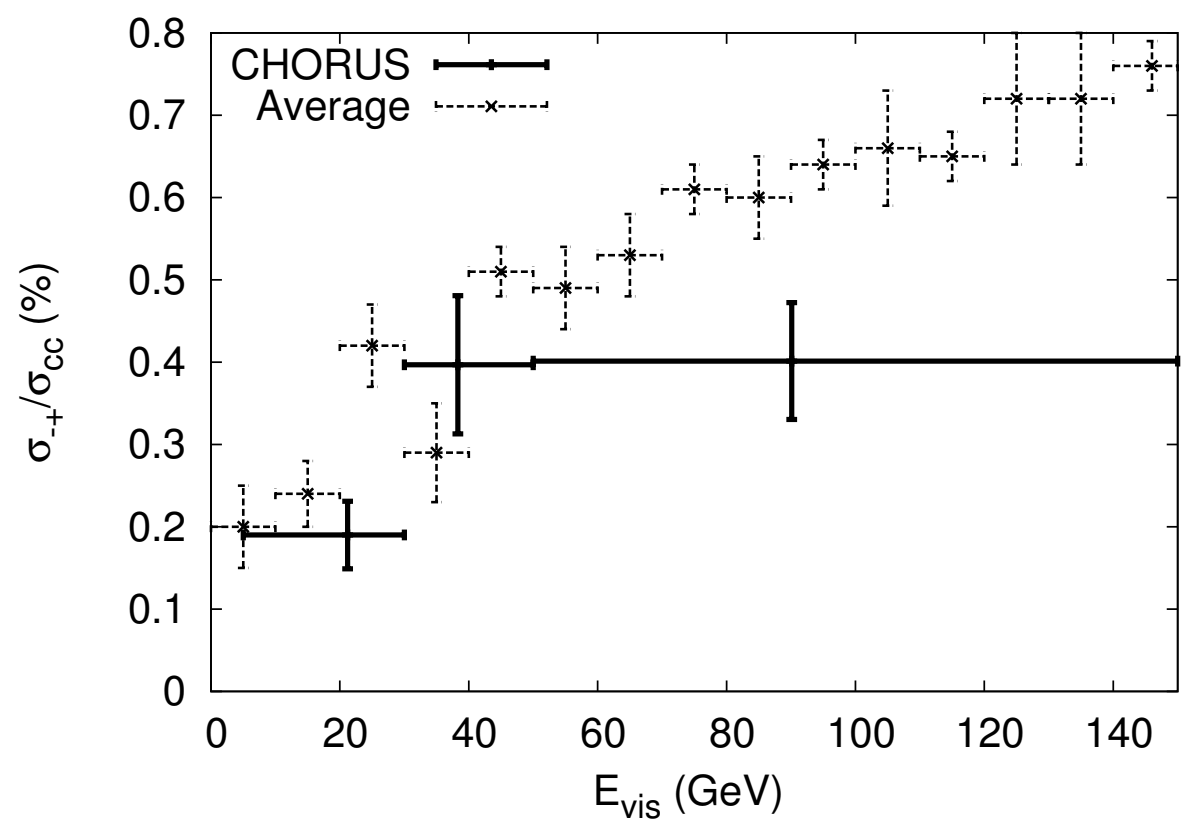

Figure 3: Dimuon neutrino cross-section normalized to the charged-current cross-section as a function of visible energy. The solid crosses show CHORUS result and the dashed ones is the average over the other dimuon experiments [36].

\section{Acknowledgements}

We gratefully acknowledge the help and support of the neutrino beam staff and of the numerous technical collaborators who contributed to the detector construction, operation, emulsion pouring, development, and scanning. The experiment was made possible by grants from the Institut Interuniversitaire des Sciences Nucléaires and the Interuniversitair Instituut voor Kernwetenschappen (Belgium), the Israel Science Foundation (grant 328/94) and the Technion Vice President Fund for the Promotion of Research (Israel), CERN (Geneva, Switzerland), the German Bundesministerium für Bildung und Forschung (Germany), the Institute of Theoretical and Experimental Physics (Moscow, Russia), the Istituto Nazionale di Fisica Nucleare (Italy), the Promotion and Mutual Aid Corporation for Private Schools of Japan and Japan Society for the Promotion of Science (Japan), the Korea Research Foundation Grant (KRF-2003005-C00014) (Republic of Korea), the Foundation for Fundamental Research on Matter FOM and the National Scientific Research Organization NWO (The Netherlands), and the Scientific and Technical Research Council of Turkey (Turkey). We gratefully acknowledge their support. 


\section{References}

[1] H. Abramowicz et al., Z. Phys. C15 (1982) 19.

[2] S.A. Rabinowitz et al., Phys. Rev. Lett. 70 (1993) 134; arXiv:hep-ex/9406007

[3] M. Jonker et al., Phys. Lett. B107 (1981) 241.

[4] P. Vilain et al., Eur. Phys. J. C11 (1999) 19.

[5] P. Astier et al., Phys. Lett. B526 (2002) 278.

[6] D. Naples et al., Nucl. Phys. (Proc. Suppl.) B118 (2003) 164; M. Goncharov et al., Phys. Rev. D64 (2001) 112006.

[7] N. Ushida et al., Phys. Lett. B206 (1988) 380; N. Ushida et al., Phys. Lett. B206 (1988) 375;

[8] T. Bolton, hep-ex/9708014, 1997.

[9] G. De Lellis et al., Phys. Rep. 399 (2004) 227.

[10] T. Nakano, Ph.D. thesis, Nagoya University, Japan (1997).

[11] A. Kayis-Topaksu et al., CHORUS Collaboration, Phys. Lett. B549 (2002) 48.

[12] B. Van de Vijver, Ph.D. thesis, Vrije Universiteit Brussel (2002).

[13] E. Eskut et al., Nucl. Instrum. and Methods A401 (1997) 7.

[14] M. Güler, Ph.D. thesis, METU, Ankara, Turkey (2000).

[15] T. Nakano, Proc. Int. Europhysics Conf. on High Energy Physics, Budapest, Hungary (2001).

[16] K. Kodama et al., Nucl. Instrum. and Methods A493 (2002) 45.

[17] N. Nonaka, Ph.D. thesis, Nagoya University, Japan (2002).

[18] G. Önengüt et al, CHORUS Collaboration, Phys. Lett. B613 (2005) 105.

[19] P. Zucchelli, Ph.D. thesis, University of Ferrara, Italy (1996).

[20] G. Ingelman, Preprint TSL/ISV 92-0065, Uppsala University, Sweden (1992).

[21] M. Sorrentino, CHORUS internal note 2000027 (2004), http://choruswww.cern.ch/Publications/Notes/charm_background.pdf

[22] P. Astier et al, NOMAD Collaboration, Nucl. Phys. B621 (2002) 3.

[23] I. Tsukerman, MC generators in CHORUS, Nucl. Phys. (Proc. Suppl.) 112 (2002) 177.

[24] S. Sorrentino, Diploma Thesis, Naples University, Italy (1995).

[25] A. Fassò et al., SARE-3 Workshop, KEK Report Proceedings 97-5 (1997) 32.

[26] F. Di Capua, Ph.D. thesis, Universitá di Napoli, Italy (2003).

[27] R.E. Shrock and B.W. Lee, Phys. Rev. D13 (1976) 2539; Erratum: Phys. Rev. D14 (1976) 313.

[28] O. Melzer, Diploma thesis, Westfälische Wilhelms-Universität, Münster, Germany (1997).

[29] GEANT 3.21, CERN program library long write up W5013.

[30] M. Glück, E. Reya and A. Vogt, Z. Phys. C67 (1995) 443.

[31] CTEQ Collaboration, H.L. Lai et al., Phys. Rev. D51 (1995) 4763.

[32] Particle Data Group, S. Eidelman et al., Physics Letters B592 (2004) 1.

[33] G.J. Feldman and R.D. Cousins, Phys. Rev. D57 (1998) 3873.

[34] K. Kodama et al, Phys. Lett. B313 (1993) 260.

[35] N. Bruski, Ph.D. thesis, Westfälische Wilhelms-Universität, Münster, Germany (2002).

[36] G. De Lellis et al., J. Phys. G: Nucl. Part. Phys. 28 (2002) 713. 